\section{Antipsychotics: A Real or Confounding Risk Factor for Venous Thromboembolism?}

\author{
C. Chapelle ${ }^{1}$, S. Quenet ${ }^{2}$, X. Delavenne ${ }^{2,3}$, K. Lacut $^{4}$, \\ P. Mismetti ${ }^{2,5,6}$, S. Laporte ${ }^{2,5}$, M. N. Beyens ${ }^{2,6}$ \\ ${ }^{1}$ INSERM CIE3, CHU Saint-Etienne, Saint-Etienne, France \\ ${ }^{2}$ EA3065, Université Jean-Monnet, Saint-Etienne, France \\ ${ }^{3}$ Laboratoire de pharmacologie et toxicologie, $\mathrm{CHU}$ \\ Saint-Etienne, Saint-Etienne, France \\ ${ }^{4}$ EA3878, Université de Bretagne Occidentale, CHU Brest, Brest, \\ France \\ ${ }^{5}$ Unité de Recherche Clinique, Innovation, Pharmacologie, $\mathrm{CHU}$ \\ Saint-Etienne, Saint-Etienne, France \\ ${ }^{6}$ Centre Régional de Pharmacovigilance, CHU Saint-Etienne, \\ Saint-Etienne, France
}

\section{Abstract \\ $\nabla$}

In a meta-analysis of case-control studies, Zhang et al. (2011) found an increased risk of venous thromboembolic events (VTE) in patients exposed to antipsychotics $(\mathrm{OR}=2.39$ [1.71-3.35]). Our updated meta-analysis including the 2 available cohort studies, recognized as a more relevant type of observational study, showed a weaker, but still strong association $(\mathrm{OR}=1.84$ $[1.39 ; 2.44])$. In view of the lack of data on the confirmed risk factors for VTE in existing studies, prospective studies including adjustment for these risk factors are warranted to confirm this association and to assess the benefit/risk ratio of antipsychotics in high-risk patients.

\section{Key words}

meta-analysis · observational studies · venous thromboembolism . antipsychotics

In 2011, Zhang et al. published in Pharmacopsychiatry a metaanalysis evaluating the association between venous thromboembolic events (VTE) and antipsychotic drug exposure [1]. The results of this analysis showed an increased risk of VTE in patients exposed to antipsychotics (odds ratio $(\mathrm{OR})=2.39$ [1.713.35]). Only case-control studies were included in the metaanalysis and the results obtained in the groups currently and recently exposed to antipsychotics, respectively, as defined in each study, were pooled regardless of the differences in the definitions of exposure between the studies. At the time of that publication, we were conducting a similar meta-analysis, but in contrast to Zhang et al., we included both case-control and cohort studies. We also checked whether the timing of exposure might modify the effect of antipsychotics on the risk of VTE.

As case-control studies are known to overestimate risks, cohort studies are necessary to validate their results [2]. For that matter, Zhang et al. noted that "additional studies in large cohorts are required to validate [their] findings". To the best of our knowledge, 2 large retrospective cohort studies focusing on this topic have been published to date [3,4]. As expected, when we included these studies, we found a weaker association between antipsychotic drug exposure and VTE risk $(\mathrm{OR}=1.84$ [1.39; 2.44], $\bigcirc$ Fig. 1).

Regarding the timing of exposure in relation to VTE, we classified patients into 2 categories: those who were taking antipsychotics when the VTE occurred or had taken antipsychotics within the previous 3 months, and those who had taken antipsychotics between 3 and 12 months prior to the event. These 2 groups were compared to unexposed patients, defined as patients who had never taken antipsychotics or had taken antipsychotics more than 1 year prior to the event. We found that the more recent the exposure, the higher the risk of VTE. The risk of VTE was increased by $85 \%$ and $65 \%$, respectively, in patients who were taking antipsychotics at the time of VTE or had taken antipsychotics within the previous 3 months and in patients who had taken antipsychotics between 3 and 12 months prior to the event ( $\mathrm{OR}=1.85[1.37 ; 2.50]$ and $\mathrm{OR}=1.65[1.51 ; 1.80]$ ) compared to unexposed patients. This is in favour of a causal relationship between antipsychotics and VTE. The meta-analysis of adjusted OR gave the same results. However, most of the known risk factors for VTE were not taken into account. The association detected could therefore also be due to confounding factors, in particular, to the presence of known risk factors for VTE. Some of these factors, such as immobilization indeed apply to patients taking antipsychotics. Nevertheless, even though our results are based solely on univariate analyses, they remain striking. It is therefore important to confirm whether or not exposure to antipsychotics is an independent risk factor for VTE. At present, this question cannot be answered by any meta-analysis because none of the studies reported to date recorded the presence of confirmed risk factors for VTE. Like Zhang et al., we emphasize the need for a new large prospective cohort study that would also collect data on the already known risk factors for VTE.

\section{Conflict of interest \\ $\nabla$}

The authors declare no conflicts of interest.

\section{Study design}

Case-control (6 studies: 188,108 patients)

Cohort ( 2 studies: 187,565 patients)

Overall (8 studies: 375,673 patients)

Heterogeneity between subgroups: $p<0.001$

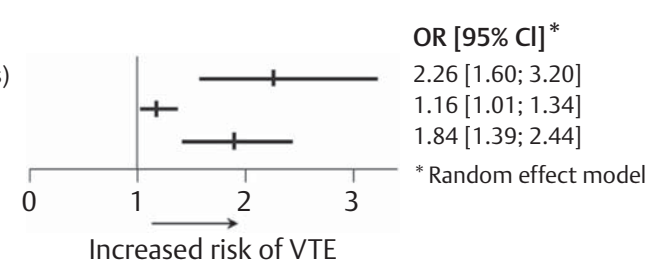

Fig. 1 risk of VTE according to study design. 


\section{References}

1 Zhang $R$, Dong $L$, Shao $F$ et al. Antipsychotics and venous thromboembolism risk: a meta-analysis. Pharmacopsychiatry $2011 ; 44: 183-188$

2 NHMRC levels of evidence and grades for recommendations for developers of guidelines. 2009; Available from: http://www.nhmrc.gov. au/_files_nhmrc/file/guidelines/evidence_statement_form.pdf

3 Ray JG, Mamdani MM, Yeo El. Antipsychotic and antidepressant drug use in the elderly and the risk of venous thromboembolism. Thromb Haemost 2002; 88: 205-209

4 Liperoti R, Pedone C, Lapane KL et al. Venous thromboembolism among elderly patients treated with atypical and conventional antipsychotic agents. Arch Intern Med 2005; 165: 2677-2682

$\begin{array}{ll}\text { received } & 07.05 .2012 \\ \text { revised } & 31.05 .2012 \\ \text { accepted } & 06.06 .2012\end{array}$

Bibliography

DOI http://dx.doi.org/

10.1055/s-0032-1316347

Published online ahead of print:

20 July 2012

Pharmacopsychiatry 2013;

46: $36-37$

(c) Georg Thieme Verlag KG

Stuttgart · New York

ISSN 0176-3679

\section{Correspondence}

\section{Celine Chapelle}

CHU Saint-Etienne

INSERM CIE3

120 Avenue Albert Raimond

42055 Saint-Etienne

\section{France}

Tel.: +33/4/7712 0283

Fax: $+33 / 4 / 77127820$

celine.chapelle@chu-st-etienne.fr 